\title{
Konsolidierungspfade der Länder und ihrer Kommunen zur Einhaltung der Schuldenbremse ab dem Jahr 2020
}

von Ingolf Deubel

Spätestens ab dem Jahr 2020 müssen alle Länder die Schuldenbremse einhalten und somit ihre Haushalte ohne neue strukturelle Schulden aufstellen und abwickeln. Gleichzeitig muss der föderale Finanzausgleich neu geordnet sein. Bei einer unterstellten Fortgeltung des jetzigen Ausgleichssystems über das Jahr 2019 hinaus könnten bis auf das Saarland und Bremen alle Länder (einschl. ihrer Kommunen) die Schuldenbremse einhalten. In einer ganzen Reihe von Ländern sind dafür allerdings noch erhebliche Konsolidierungsanstrengungen erforderlich. Die vielfach geforderte generelle Absenkung der Ausgleichsintensität des Finanzausgleichs ist unter diesen Umständen mit der verfassungsrechtlichen Vorgabe ausgeglichener Haushalte nicht zu vereinbaren. Eine Steuerautonomie der Länder ist zur Stärkung des Föderalismus wünschenswert, aber politisch allenfalls dann umsetzbar, wenn durch den Finanzausgleich sichergestellt wird, dass vor allem die steuerschwächeren neuen Länder ihre Steuersätze absenken und trotzdem die Schuldenbremse einhalten können.

By no later than 2020, all sixteen German states will have to comply with the so called "debt brake" requiring them to run their budgets without structural deficits. At the same time, there needs to be a reorganization of the federal fiscal equalization system. If the current system was continued after 2019, all of the states (and their local governments), with the exception of Saarland and Bremen, would be able to comply with the debt brake. In a number of states, however, a significant amount of consolidation efforts is still required to achieve this. Under these circumstances the frequently requested general reduction of the compensation intensity cannot be reconciled with the constitutional requirement of a balanced budget. Giving the states more autonomy in tax matters would be a desirable way to strengthen federalism, but it would only be politically feasible if the fiscal equalization can guarantee that the new states in the East with weaker tax revenues can still lower their tax rates while remaining in compliance with the debt brake.

\section{Die Vorgaben der Schuldenbremse für die Länderfinanzen}

Bund und Länder sowie die Länder untereinander haben sich im Jahr 2001 über das Maßstäbegesetz, die Neufassung des Finanzausgleichsgesetzes, den Solidarpakt II und die Ausfinanzierung des Fonds Deutsche Einheit geeinigt. Die Ver- 
einbarungen waren damals - vor allem aufgrund entsprechender Forderungen der Geberländer - nur mit einer zeitlichen Befristung der entsprechenden Gesetze bis zum 31.12.2019 möglich.

Ähnliches gilt auch für die im Jahr 2009 in Art. 143d GG vereinbarten Übergangsregeln zur Einführung der Schuldenbremse für die Länder und die Konsolidierungshilfen für die höher verschuldeten Länder. Die Konsolidierungshilfen für die Länder Bremen, Saarland, Berlin, Sachsen-Anhalt und SchleswigHolstein im Umfang von jährlich 800 Mio. Euro werden nur für den Zeitraum von 2011 - 2019 gewährt und die Schuldenbremse nach Art. 109 Abs. 3 GG wird erst im Jahr 2020 für alle Länder rechtsverbindlich. ${ }^{1}$

Mit dem Jahr 2019 laufen schließlich auch noch die im Entflechtungsgesetz aus dem Jahr 2006 (letzte Fortschreibung am 15.7.2013) geregelten investiv gebundenen Bundesmittel nach Art. 143c GG in Höhe von jährlich 2,57 Mrd. Euro aus.

Bund und Länder sind deshalb gefordert, rechtzeitig vor dem 1.1.2020 ihrer verfassungsrechtlichen Verpflichtung nachzukommen und eine den Vorgaben des Grundgesetzes genügende Neuordnung des föderalen Finanzausgleichs vorzunehmen. Die verfassungsrechtliche Vorgabe des Art. 107 Abs. 2 GG lautet: Sicherstellung eines angemessenen Ausgleichs der unterschiedlichen Finanzkraft der Länder mit Berücksichtigung der Finanzkraft und des Finanzbedarfs der Gemeinden. Weitere Präzisierungen finden sich in verschiedenen Artikeln des Grundgesetzes (Art 28, 72, 106, 109 und 143).

Im Ergebnis muss der föderale Finanzausgleich nach seiner Neuordnung somit sicherstellen, dass alle Länder einschließlich ihrer Kommunen ab dem Jahr 2020 prinzipiell in der Lage sind ihre Haushalte in konjunkturellen Normallagen ohne neue Kredite auszugleichen. Der Begriff ,angemessener Ausgleich“ ist dabei so zu interpretieren, dass der Ausgleich mindestens so stark sein muss, dass auch steuerschwächere Länder (einschließlich ihrer Kommunen) bei entsprechender Sparsamkeit die Schuldenbremse einhalten können. Es darf dabei allerdings nach der Rechtsprechung des BVerfG nicht zu einer Nivellierung oder gar einer Umkehr der Finanzkraftreihenfolge kommen. Aus ökonomischer Sicht sollten darüber hinaus hinreichende Anreize zur Pflege und Entwicklung der eigenen Steuerquellen verbleiben.

1 Vgl.: Deubel, I.: Die Föderalismusreform II: eine sinnvolle Weiterentwicklung der Verschuldungsgrenzen, in: Zeitschrift für Staats- und Europawissenschaften, 2/2009, 231 - 249, hier $240 \mathrm{ff}$. 
Damit sich der Bund und die Länder (einschließlich der Kommunen) rechtzeitig auf die ab dem Jahr 2020 geltenden Regeln vorbereiten können, erscheint es notwendig, eine entsprechende Einigung zwischen Bund und Ländern noch in der laufenden Legislaturperiode des Bundestags - also spätestens bis zum Sommer 2017 - zu erreichen.

Nicht auszuschließen ist es dabei natürlich, dass es auch zu Veränderungen des Art. 107 GG kommt. Dies gilt insbesondere für die Vorgaben zur Zerlegung der Steuern und zur Verteilung des Länderanteils an der Umsatzsteuer. Mit einiger Sicherheit dürfte dabei auch erneut in intensiver Form über die Frage von $\mathrm{Zu}$ schlagsrechten auf der Länderebene für die Einkommen- und Körperschaftsteuer diskutiert werden. ${ }^{2}$

Die Diskussion über die Neuordnung der föderalen Finanzbeziehungen zwischen Bund, Ländern und Gemeinden kann nicht unabhängig von der realen Finanzsituation der Länder und ihrer Gemeinden erfolgen. In diesem Beitrag soll deshalb untersucht werden, in welcher relativen und absoluten Finanzsituation sich die einzelnen Länder einschließlich ihrer Kommunen im Jahr 2020 bei unverändertem Finanzausgleich befinden dürften und ob sie aus heutiger Sicht zur Einhaltung der Schuldenbremse in der Lage sein werden.

Um diese Frage zu beantworten, wird zunächst im Kapitel II analysiert, welche Finanzmassen die einzelnen Länder und ihre Kommunen im Jahr 2013 in ihren Kern- und Extrahaushalten in Anspruch genommen haben und welche Bedeutung die Zinsen, Versorgungsausgaben, Sachinvestitionen und Sozialausgaben für die Finanzsituation der einzelnen Länder haben. ${ }^{3}$ Unter der verfügten Finanzmasse wird dabei die Summe aus den eigenen Steuereinnahmen, den erhaltenen Zahlungen von anderen Ländern (abzüglich der Zahlungen an andere Länder), vom Bund und der EU abzüglich des Finanzierungssaldos und des Saldos der finanziellen Transaktionen verstanden.

Auf der Basis der Ausgangssituation des Jahres 2013 werden im anschließenden Kapitel III die im Jahr 2020 voraussichtlich verfügbaren absoluten und relativen Finanzmassen der einzelnen Länder einschließlich ihrer Kommunen projiziert

2 Vgl. z.B. Feld, L.P./ Kube, H./ Schnellenbach, J.: Optionen für eine Reform des bundesdeutschen Finanzausgleichs, Freiburg, 2013, $28 \mathrm{ff}$.

3 Die in den Kapiteln II und III angewendete Methodik erfolgt in Anlehnung an: Detemple, P./ Michels, Y./ Schramm, Th.: PwC-Länderfinanzbenchmarking, 2014, 38 - 70 und 209 - 216. Die Studie von PwC beschränkt sich allerdings im Wesentlichen auf die Kernhaushalte und verzichtet auf eine Bereinigung um den Saldo der finanziellen Transaktionen. 
und dabei auch die besondere Bedeutung der Belastungen aus Zinsen und Versorgungsleistungen herausgearbeitet.

Dabei wird sich zeigen, dass die zur Einhaltung der Schuldenbremse im Jahr 2020 erforderlichen Konsolidierungspfade für einige Länder sehr ambitioniert sind, sodass erhebliche Zweifel an ihrer Realisierbarkeit entstehen. Dies gilt erst recht, wenn alternative Risikoszenarien mit einem geringeren Wachstum der Steuereinnahmen oder einem höheren Zinsniveau unterstellt werden.

Im abschließenden Kapitel IV wird deshalb dargestellt, welche Schlussfolgerungen sich aus diesen Ergebnissen für die anstehende Neuordnung des föderalen Finanzausgleichs ziehen lassen.

\section{Die Finanzen der Länder und ihrer Kommunen im Jahr 2013}

\section{Die in Anspruch genommenen Finanzmassen}

Für bestimmte Fragestellungen kann es durchaus sinnvoll sein, die Ausgabenund Einnahmenstrukturen der Kernhaushalte der einzelnen Länder sowie der Kommunen im Detail zu analysieren. Bei Vergleichen zwischen den Ländern wird allerdings schnell deutlich, dass die unterschiedlichen Privatisierungs-, Kommunalisierungs- und Kostendeckungsgrade eine Vergleichbarkeit einzelner Ausgaben- und Einnahmenpositionen stark einschränken und etliche Länder große Teile ihrer Aufgaben (wie z.B. den Betrieb der Hochschulen oder das Immobilien- und Straßenmanagement) gar nicht mehr in den Kern-, sondern in Extrahaushalten wahrnehmen.

Ein großer Teil der dadurch entstehenden Vergleichsstörungen lässt sich durch eine konsolidierte Betrachtung der Länder und ihrer Kommunen einschließlich der Extrahaushalte beheben, aber es bleiben auch dann noch die sich aus unterschiedlichen Privatisierungsgraden ergebenden Probleme bestehen.

So taucht z.B. eine private Kindertagesstätte im Haushalt der Kommune im Regelfall nur mit ihrem Zuschuss aus öffentlichen Geldern auf, nicht jedoch mit den gesamten Ausgaben und den unmittelbaren eigenen Einnahmen der privaten Kindertagesstätte aus Gebühren und Entgelten und auch nicht mit dem eventuellen Zuschuss des privaten Trägers. Um eine - aus der Sicht des kommunalen Haushalts - sinnvolle Vergleichbarkeit herzustellen, ist es deshalb ratsam, auf das Konzept des Zuschussbedarfs der eingesetzten bzw. der verfügbaren Finanzmassen abzustellen. Unter der verfügbaren Finanzmasse wird dabei die Summe aus den eigenen Steuereinnahmen, den Zuweisungen und Zuschüssen 
vom Bund, der EU und von anderen Ländern (abzüglich der Zahlungen an andere Länder) verstanden, wobei die Haushalte der Länder und ihrer Kommunen konsolidiert werden und die Extrahaushalte einbezogen sind. Die tatsächlich eingesetzte Finanzmasse entspricht der so definierten verfügbaren Finanzmasse abzüglich des Finanzierungssaldos und des Saldos der finanziellen Transaktionen.

Im Durchschnitt der alten Flächenländer und ihrer Kommunen betrug im Jahr 2013 die verfügte Finanzmasse 4.182 Euro und die verfügbare Finanzmasse 4.202 Euro pro Einwohner. Diese Werte werden jeweils auf $100 \%$ normiert. Alternativ könnte auch der Durchschnitt aller Länder (4.349 Euro bzw. 4.417 Euro pro Einwohner) zur Normierung verwendet werden. Dagegen spricht allerdings zum einen, dass die neuen Länder aus dem Solidarpakt II noch erhebliche zusätzliche Mittel erhalten und diese bis 2020 weitgehend weggefallen sein werden und zum anderen, dass sich auch im horizontalen Finanzausgleich der Bedarfswert von $100 \%$ nur auf die Flächenländer bezieht und den Stadtstaaten ein Bedarfswert von $135 \%$ zugestanden wird.

Insofern spricht einiges dafür, die Normierung am Durchschnitt der alten Flächenländer zu orientieren. Insbesondere bei der Interpretation der Entwicklung zwischen 2013 und 2020 ergibt sich auf diese Weise eine bessere Vergleichbarkeit.

Die Reihenfolge der Länder in der Abbildung 1 orientiert sich an der verfügten Finanzmasse. Bremen liegt hier mit 155,6 \% weit vor allen anderen Ländern und wird gefolgt von Hamburg mit 144,1 \% und Berlin mit 137,7 \%. Bremen kann zwar aufgrund der Konsolidierungshilfe von 300 Mio. Euro mit 143,1 \% zugleich auch über die höchste Finanzmasse aller Länder verfügen, die Differenz zwischen tatsächlicher Verfügung und Verfügbarkeit beträgt jedoch 12,5 Punkte. Auch in Hamburg ergibt sich eine Lücke von 6,9 Punkten, während Berlin einen Überschuss von 4,4 Punkten aufweist.

Unter den Flächenländern nimmt das Saarland mit 117,9 \% die höchste Finanzmasse in Anspruch, verfügbar sind jedoch nur 98,4 \%. Mit 19,5 Punkten ist der absolute Abstand somit noch deutlich höher als in Bremen. Die zweithöchste relative Überschreitung unter den Flächenländern weist Hessen mit 6,1 Punkten auf. Danach folgen Nordrhein-Westfalen mit 2,2 Punkten und Rheinland-Pfalz mit 1,9 Punkten. 
Abbildung 1: Verfügte und verfügbare Finanzmassen pro Einwohner der Länder und ihrer Kommunen im Jahr 2013 in Relation zum Durchschnitt der alten Flächenländer

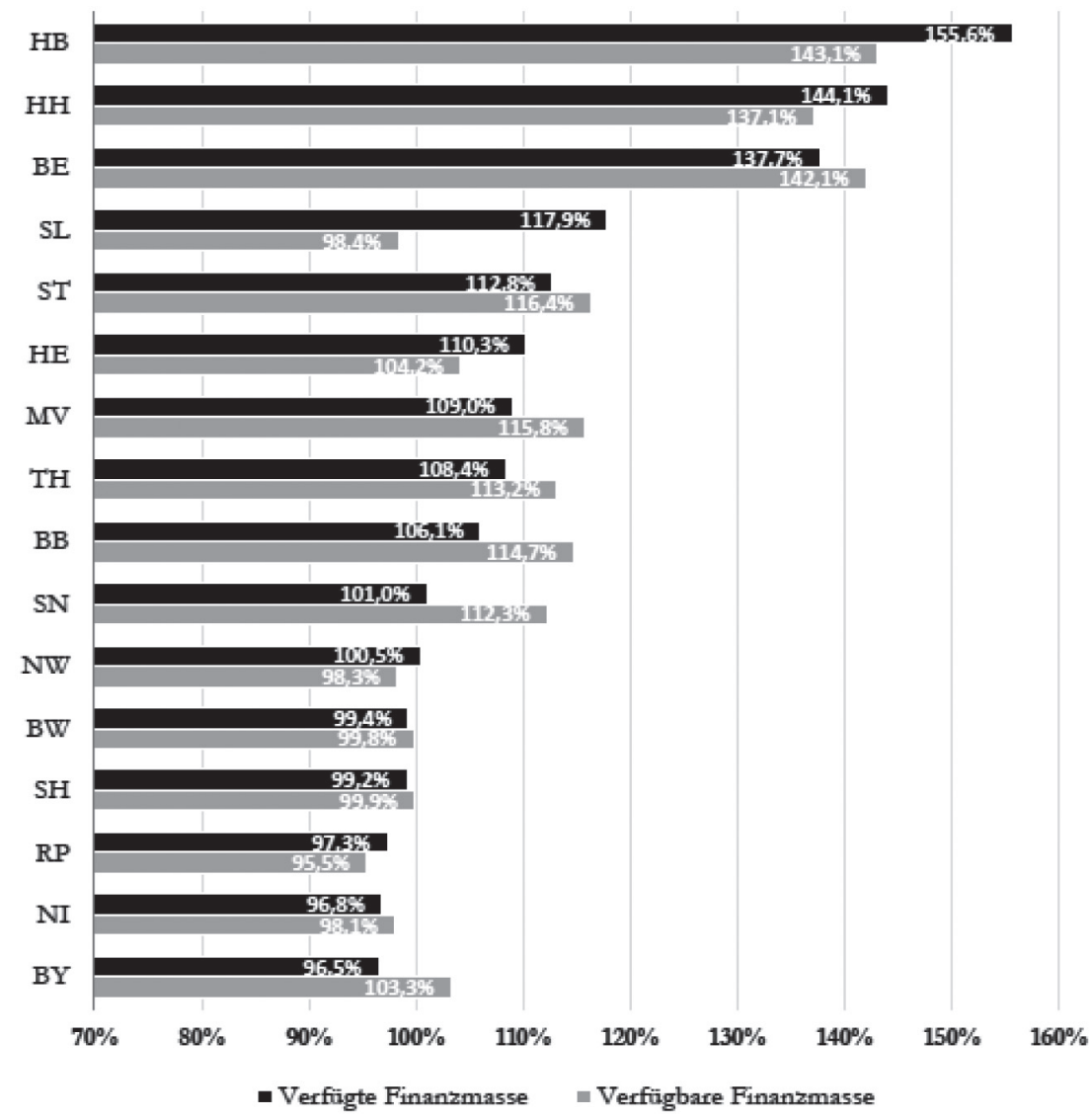

Quellen: Statistisches Bundesamt (Fachserie 14 Reihe 2, Vierteljährliche Kassenergebnisse des öffentlichen Gesamthaushalts, 1. - 4. Vierteljahr 2013 einschließlich Auslaufperiode) und eigene Berechnungen.

Bayern nimmt mit 96,5 \% die geringste Finanzmasse aller Länder in Anspruch, gefolgt von Niedersachsen mit 96,8 \% und Rheinland-Pfalz mit 97,3\%. Da Bayern mit 103,3 \% unter den alten Flächenländern zugleich über die höchste Finanzmasse verfügen kann, liegen die verfügten Mittel um 6,8 Punkte unter den verfügbaren Mitteln. Auch in Niedersachsen ergibt sich noch ein „Sicherheitsabstand" von 1,2 Punkten, während Rheinland-Pfalz als das Land mit der gerings- 
ten verfügbaren Finanzmasse aller 16 Länder noch eine Lücke von 1,9 Punkten aufweist. Dagegen erwirtschaften Schleswig-Holstein und Baden-Württemberg trotz ihrer höheren in Anspruch genommenen Finanzmassen noch Überschüsse von 0,8 Punkten bzw. 0,4 Punkten.

Völlig anders ist die Situation (noch) in den neuen Ländern. Hier liegen die verfügbaren Finanzmassen zwischen 112,3\% in Sachsen und 116,4 \% in SachsenAnhalt und die in Anspruch genommenen Mittel zwischen 101,0\% in Sachsen und 112,8 \% in Sachsen-Anhalt. Daraus ergibt sich für Sachsen mit 11,2 Punkten der mit Abstand höchste Überschuss aller Länder. Dahinter folgen Brandenburg mit 8,7 Punkten, Mecklenburg-Vorpommern mit 6,8 Punkten, Thüringen mit 4,8 Punkten und Sachsen-Anhalt mit 3,6 Punkten.

\section{Die für Zinsen und Versorgung eingesetzten Mittel}

Bei der Gesamtbetrachtung wird nicht berücksichtigt, dass ein Teil der verfügbaren Mittel bereits vorab zur Finanzierung von „Altlasten“ aus Zinsen und Versorgung gebunden ist und sich diese Belastungen keineswegs gleichmäßig auf die Länder verteilen, sondern sehr unterschiedliche Niveaus aufweisen.

Unter Versorgung werden dabei die Versorgungslasten für Pensionäre und Hinterbliebene, die Beihilfe für Versorgungsempfänger und die Zahlungen der neuen Länder nach dem AAÜG verstanden. Nach dem Gesetz zur Überführung der Ansprüche und Anwartschaften aus Zusatz- und Sonderversorgungssystemen des Beitrittsgebiets (AAÜG) müssen die neuen Länder dem Bund seit dem Jahr 2010 einen Anteil von $60 \%$ dieser Aufwendungen erstatten. Faktisch handelt es sich dabei um Leistungen, die mit einer Beamtenversorgung vergleichbar sind, aber in die Rentenversicherung überführt wurden. 2013 betrugen die Zahlungen der neuen Länder (einschließlich Berlin) an den Bund nach dem AAÜG fast 2,6 Mrd. Euro. Nach Berechnungen von Seitz ${ }^{4}$ dürften die Zahlungen aus dem AAÜG in den Jahren 2016 oder 2017 ihr Maximum erreicht haben und sich danach entsprechend der Verteilung der Lebenserwartung langsam reduzieren.

Mit 982 Euro/E hat das Land Bremen mit Abstand die höchsten Zinslasten, gefolgt vom Saarland mit 577 Euro/E, Berlin mit 565 Euro/E, Hamburg mit 496 Euro/E, Nordrhein-Westfalen mit 356 Euro/E und Schleswig-Holstein mit 355 Euro/E. Am Ende liegen Sachsen mit 105 Euro/E, Bayern mit 117 Euro/E und Brandenburg mit 241 Euro/E.

4 Seitz, H.: Fortschreibung der Projektion über die Zahlungsverpflichtungen des Freistaats Sachsen und der anderen ostdeutschen Länder aus dem AAÜG, Berlin, 2008. 
Abbildung 2: Verfügbare und verfügte Finanzmasse nach Zinsen und Versorgung in Euro/E im Jahr 2013

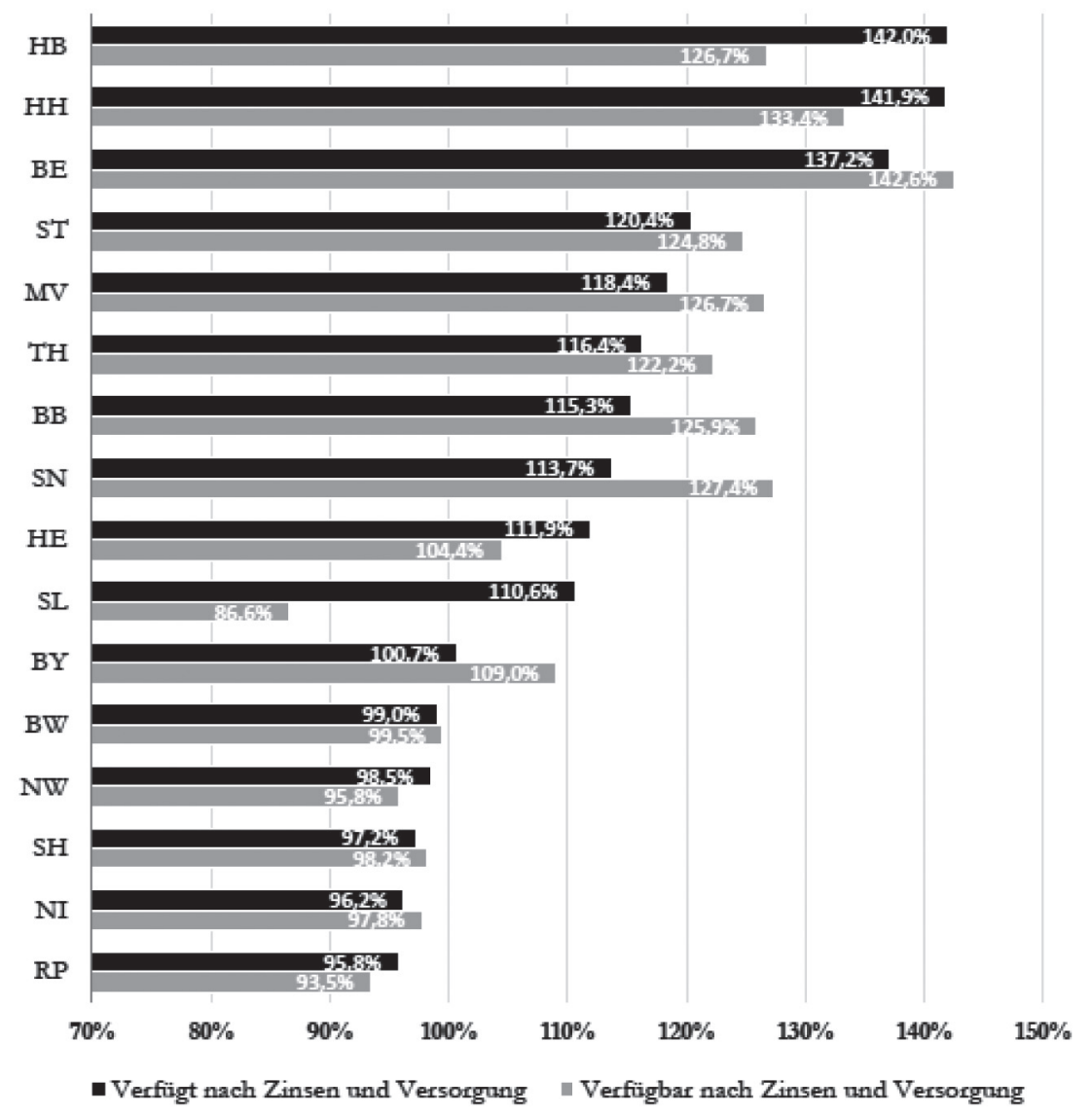

Quellen: Statistisches Bundesamt (Fachserie 14 Reihe 2) und eigene Berechnungen

Bei den Ausgaben für Versorgung liegen die neuen Flächenländer mit Belastungen zwischen 268 Euro/E in Sachsen und 314 Euro in Sachsen-Anhalt trotz der Zahlungen aus dem AAÜG weit hinter den alten Ländern, bei denen Ausgaben zwischen 486 Euro/E in Niedersachsen und 605 Euro/E im Saarland entstehen. Bei den Stadtstaaten trägt Hamburg mit 721 Euro/E die höchste Belastung, gefolgt von Bremen mit 713 Euro/E und Berlin mit 545 Euro/E.

Die besonders hohen Belastungen des Saarlandes beruhen u.a. auch sehr stark auf dem erheblichen Rückgang der Einwohnerzahlen, so dass die Versorgungs- 
lasten zu immer größeren Ausgaben pro Einwohner führen. Diese Kostenremanenz, die rein rechnerisch natürlich auch für die Zinslasten entsteht, betrifft bei den alten Flächenländern nur das Saarland und bei den neuen Flächenländern vor allem Sachsen-Anhalt, Thüringen und Mecklenburg-Vorpommern.

Von daher erscheint es zunächst einmal durchaus nachvollziehbar, wenn etliche hoch verschuldete und defizitär wirtschaftende Länder die Behauptung aufstellen, dass ihre Finanzprobleme vor allem auf die „Altlasten“ aus Zinsen und Versorgung zurückzuführen seien.

Insbesondere beim Saarland und bei Bremen ist jedoch festzustellen, dass auch die in Anspruch genommene Finanzmasse nach Zinsen und Versorgung sich noch weit oberhalb der vergleichbaren Länder bewegt. Das Saarland liegt mit $110,6 \%$ auch hier noch weit über dem Durchschnitt der alten Flächenländer, wobei nunmehr allerdings Hessen mit 111,9 \% die höchste Inanspruchnahme der alten Flächenländer aufweist. Bremen liegt mit 142,0 \% knapp über Hamburg mit 141,9 \%, aber deutlich vor Berlin mit 137,2 \%. Unter den Flächenländern am sparsamsten sind Rheinland-Pfalz mit 95,8\%, Niedersachsen mit 96,2\% und Schleswig-Holstein mit 97,2\%.

\section{Die für Sachinvestitionen eingesetzten Mittel}

Die relative Verbesserung von Bremen und der Positionswechsel zwischen Hessen und dem Saarland beruhen allerdings auch darauf, dass im Saarland und in Bremen extrem niedrige Sachinvestitionen vorgenommen werden, während Hamburg 2013 die höchsten Sachinvestitionen aller Länder vornahm und Hessen zumindest überdurchschnittlich investierte.

Zieht man nämlich auch noch die Sachinvestitionen ab und berechnet die verfügte Finanzmasse ohne Zinsen, Versorgung und Sachinvestitionen, so liegt Bremen mit 154,1 \% wieder deutlich vor Hamburg mit 137,2 \% und das Saarland weist mit 116,7 \% erneut den höchsten Wert der alten Flächenländer auf.

Beim Ländervergleich der Sachinvestitionen ergeben sich nämlich geradezu dramatische Unterschiede zwischen 724 Euro/E beim Spitzenreiter Hamburg und 181 Euro/E beim Schlusslicht Berlin. Aber auch Bremen mit 226 Euro/E, Nordrhein-Westfalen mit 261 Euro/E und das Saarland mit 276 Euro/E weisen extrem niedrige Sachinvestitionen auf. 
Abbildung 3: Verfügbare und verfügte Finanzmasse nach Zinsen, Versorgung und Sachinvestitionen im Jahr 2013 in Euro/E

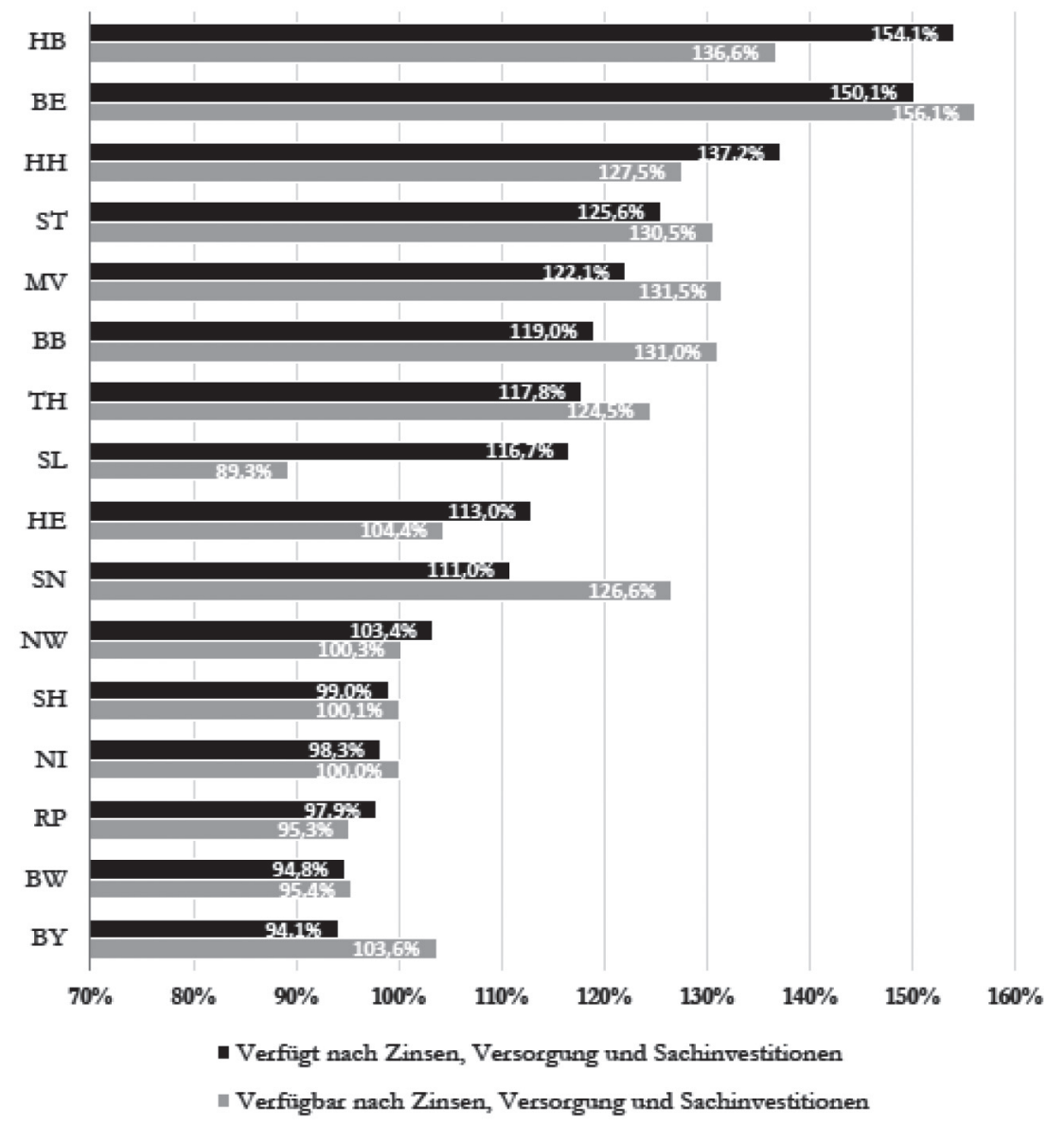

Quellen: Statistisches Bundesamt (Fachserie 14 Reihe 2) und eigene Berechnungen

Auf der anderen Seite liegen die Sachinvestitionen nicht nur in Hamburg, sondern auch in Bayern mit 611 Euro/E, Sachsen mit 550 Euro/E, BadenWürttemberg mit 534 Euro/E, Thüringen mit 436 Euro/E und Hessen mit 431 Euro/E über dem Durchschnitt von 411 Euro/E.

Um einen Maßstab zur Beurteilung der Angemessenheit der Sachinvestitionen zu haben, wäre es notwendig, für jedes Land auch die Abschreibungen darzustellen. Diese werden in der Kameralistik allerdings nicht erfasst und die amtliche Statistik liefert auch keine anderen nach Ländern aufgeschlüsselten Daten. Erfasst 
werden die bundesweiten Abschreibungen nur in der volkswirtschaftlichen Gesamtrechnung und sind dort nicht nach Ländern, sondern nur nach Ebenen differenziert. In 2013 lagen nach der VGR die Abschreibungen auf das Sachkapital der Länder und ihrer Kommunen bundesweit bei 456 Euro/E, die Sachinvestitionen jedoch nur bei 411 Euro/E. Von daher hat sich das Sachkapital der Länder und ihrer Kommunen in 2013 um ca. 45 Euro/E bzw. rund 3,6 Mrd. Euro verringert.

Lediglich in Hamburg, Bayern, Sachsen und Baden-Württemberg dürfte es in 2013 noch zu einem Zuwachs an Sachkapital gekommen sein, während es (bei einer unterstellten Gleichverteilung des Infrastrukturbestands) in den anderen Ländern zu einem teilweise dramatischen Abbau gekommen sein dürfte.

\section{Die für Sozialleistungen eingesetzten Mittel}

Als weiterer Grund für eine hohe in Anspruch genommene Finanzmasse werden sehr häufig überdurchschnittliche Sozialausgaben aufgrund ungünstiger Wirtschafts- und Sozialstrukturen genannt. Die wichtigsten sozialen Ausgaben der Länder und ihrer Kommunen sind die Kosten der Unterkunft nach dem SGB II, die Eingliederungshilfe für behinderte Menschen, die sonstige Sozialhilfe und die Belastungen aus der Jugendhilfe. Im Folgenden werden diese sozialen Lasten näher betrachtet und untersucht, ob sich die Unterschiede zwischen den Ländern auf die Unterschiede der Wirtschafts- und Sozialstrukturen zurückführen lassen.

Während die Kosten der Unterkunft nach dem SGB II von der Bundesagentur für Arbeit sehr zeitnah dokumentiert werden, können die Nettoausgaben für die übrigen sozialen Leistungen nur der nach einzelnen Aufgaben gegliederten Rechnungsstatistik oder den jeweiligen Fachstatistiken entnommen werden. Im Juli 2014 liegt die Rechnungsstatistik jedoch nur bis zum Jahr 2011 vor und die Ergebnisse der Fachstatistiken nur bis zum Jahr 2012.

Um auf der Basis der Fachstatistik zu einer vergleichbaren Darstellung für das Jahr 2013 zu kommen, wird deshalb unterstellt, dass die Nettoausgaben in den einzelnen Leistungen jeweils die gleiche Steigerungsrate aufweisen wie die Nettoausgaben des Jahres 2012 gegenüber dem Jahr 2011. Da in den einzelnen Ländern im Zeitablauf beachtliche Schwankungen der Zuwachsraten auftreten, werden die jeweiligen Nettoausgaben der einzelnen Länder und ihrer Kommunen des Jahres 2012 mit den bundesweiten Zuwachsraten des Jahres 2012 fortgeschrieben. Für die Eingliederungshilfe lag die Zuwachsrate bei 5,8 \%, für die sonstige Sozialhilfe bei $4,9 \%$ und die Jugendhilfe bei 7,0 \%. 
Die hohe Steigerungsrate der Jugendhilfe beruht dabei vor allem auf dem weiter anhaltenden Ausbau der Kindertagesstätten (rund 55 \% der Nettoausgaben), zum Teil aber auch auf den nach wie vor steigenden übrigen Leistungen nach dem SGB VIII (rund $45 \%$ der Nettoausgaben).

Die hier erfassten und hochgerechneten Nettoausgaben für soziale Leistungen im Jahr 2013 liegen bundesweit insgesamt bei 70,3 Mrd. Euro bzw. 873 Euro/E. Davon entfielen auf die Kosten der Unterkunft 13,8 Mrd. Euro, auf die Eingliederungshilfe 14,5 Mrd. Euro, auf die sonstige Sozialhilfe 10,7 Mrd. Euro und auf die Jugendhilfe 31,2 Mrd. Euro.

Der Bund beteiligt sich in fühlbarer Weise vor allem an den Kosten der Unterkunft, wo er 2013 mit 5,0 Mrd. Euro rund 36,4\% der Kosten trug und an der Grundsicherung im Alter und bei Erwerbsminderung, wo er im vergangenen Jahr $75 \%$ und ab dem Jahr 2014 sogar $100 \%$ der Nettoausgaben von bundesweit rund 5 Mrd. Euro erstattet. Diese Zahlungen des Bundes sind in der in diesem Beitrag vorgenommenen Abgrenzung der Finanzmasse auch Bestandteil der verfügbaren Mittel der Länder und ihrer Kommunen.

Darüber hinaus beabsichtigt der Bund ab dem Jahr 2018 die Kommunen im Rahmen der Verabschiedung des Bundesteilhabegesetzes im Umfang von fünf Milliarden Euro jährlich von der Eingliederungshilfe zu entlasten. Bereits im Vorgriff auf das geplante Bundesteilhabegesetz sollen die Kommunen in den Jahren 2015 bis 2017 um jeweils 1 Mrd. Euro - jeweils hälftig durch einen höheren Bundesanteil an den Kosten der Unterkunft und einen höheren Anteil der Gemeinden an der Umsatzsteuer - entlastet werden ${ }^{5}$.

Während die Flächenländer im Jahr 2013 im Durchschnitt mit Nettoausgaben für soziale Leistungen von 832 Euro/E belastet werden, sind es in Berlin 1.443 Euro/E, in Bremen 1.356 Euro/E und in Hamburg 1.315 Euro/E. Unter den Flächenländern weisen Nordrhein-Westfalen mit 991 Euro, das Saarland mit 972 Euro/ und Hessen mit 940 Euro/E die höchsten Ausgaben aus. Die geringsten Belastungen finden sich dagegen in Baden-Württemberg mit 663 Euro/E und Bayern mit 699 Euro/E. Das Mittelfeld bilden die neuen Länder mit Belastungen zwischen 736 Euro/E in Thüringen und 871 Euro/E in Brandenburg und die übrigen alten Flächenländer Rheinland-Pfalz mit 842 Euro/E, SchleswigHolstein mit 852 Euro/E und Niedersachsen mit 853 Euro/E.

5 Vgl.: $B M F$ : Kurzfassung - Kabinettvorlage zum Regierungsentwurf zum Bundeshaushalt 2015 und Finanzplan bis 2018, abrufbar unter: http://www.bundesfinanzministerium.de/Content/DE/ Downloads/Abt_2/2014-07-02-BHH2015-Kurzfassung.pdf?_blob=publicationFile\&v=3 
Die drei Stadtstaaten liegen mit ihren Nettoausgaben für jede einzelne der vier Leistungsarten deutlich oberhalb des Durchschnitts der Flächenländer und belegen sowohl bei den Kosten der Unterkunft, als auch der sonstigen Sozialhilfe und der Jugendhilfe jeweils die ersten drei Plätze. Auch bei der Eingliederungshilfe weist mit Bremen ein Stadtstaat mit 257 Euro/E die bei weitem höchsten Nettoausgaben aus. Dahinter rangieren jedoch mit Nordrhein-Westfalen (218 Euro/E) und Niedersachsen (213 Euro/E) zwei Flächenländer, erst danach folgt Hamburg mit 211 Euro/E, knapp gefolgt von Schleswig-Holstein mit 209 Euro/E, während Berlin mit 205 Euro/E nur die fünfhöchste Belastung aufweist.

Beschränkt man die Betrachtung auf die 13 Flächenländer, so fällt auf, dass es bei den vier Leistungsarten völlig unterschiedliche Rangfolgen gibt. Bei den Kosten der Unterkunft weisen Mecklenburg-Vorpommern und Sachsen-Anhalt die höchsten Nettoausgaben auf, bei der Eingliederungshilfe NordrheinWestfalen und Niedersachsen, bei der sonstigen Sozialhilfe und der Jugendhilfe das Saarland und Hessen.

Die niedrigsten Nettoausgaben für die Kosten der Unterkunft finden sich in Bayern und Baden-Württemberg, für die Eingliederungshilfe in Sachsen und BadenWürttemberg, für die sonstige Sozialhilfe in Sachsen und Thüringen und die Jugendhilfe in Schleswig-Holstein und Niedersachsen.

Dieser Befund verdeutlicht, dass es keineswegs so ist, dass die Unterschiede in den Nettoausgaben für die vier Leistungsarten sich auf die gleiche Ursache, nämlich entsprechende Unterschiede in den Wirtschafts- und Sozialstrukturen, zurückführen lassen, sondern dass hier möglicherweise ganz andere Faktoren eine Rolle spielen. Es lässt sich nämlich zeigen, dass die Quote der Arbeitslosen pro Einwohner als wichtigster Indikator für die Unterschiede in den Wirtschaftsund Sozialstrukturen bei einer Betrachtung der Gesamtheit der Flächenländer nur mit den Kosten der Unterkunft korreliert ist, nicht dagegen mit den anderen drei Leistungsarten.

Die Streuung der Kosten der Unterkunft lässt sich zu 88,2 \% (Korrelationskoeffizient $=+0,94$ ) durch die Unterschiede der Arbeitslosenquoten an der Bevölkerung erklären. Dieser hochsignifikante Wert ist deshalb bemerkenswert, weil es zwischen den Ländern zum einen Unterschiede in der Struktur der Arbeitslosigkeit und zum anderen große Unterschiede der Mietpreisniveaus gibt.

Die Korrelationskoeffizienten der Arbeitslosenquote mit den übrigen Kostenarten betragen - 0,35 für die sonstige Sozialhilfe, - 0,21 für die Eingliederungshilfe 
und $+0,02$ für die Jugendhilfe. Bei einer Irrtumswahrscheinlichkeit von $5 \%$ wäre eine statistische Signifikanz jedoch erst bei Korrelationskoeffizienten mit einem Absolutwert von mehr als 0,55 gegeben. Hinzu kommt, dass die negativen Korrelationskoeffizienten der Arbeitslosenquote mit der sonstigen Sozialhilfe und der Eingliederungshilfe unplausibel sind. Die Unterschiede zwischen den Ländern lassen sich deshalb kaum auf unterschiedlichen Arbeitslosenquoten zurückführen. Es drängt sich vielmehr der Verdacht auf, dass die Unterschiede eher auf autonomen Entscheidungen der einzelnen Länder und ihrer Kommunen beruhen als auf objektiven Strukturmerkmalen.

In den Flächenländern insgesamt stehen deshalb nur die Kosten der Unterkunft (als bundeseinheitlich normiertes Geldleistungsgesetz) in einem sehr engen und hochsignifikanten Zusammenhang zur Wirtschafts- und Sozialstruktur, nicht jedoch die Eingliederungshilfe, die sonstige Sozialhilfe und die Jugendhilfe. Eine Beteiligung des Bundes an den sozialen Kosten erscheint deshalb nur bei normierten Geldleistungsgesetzen sinnvoll, nicht jedoch bei Sachleistungsgesetzen mit hohen Autonomiegraden der einzelnen Länder und ihrer Kommunen.

Aufgrund der föderalen Vielfalt würde eine hinreichende bundesweite Standardisierung bei Sachleistungsgesetzen wohl nur dann erreicht, wenn der Bund auch die Aufgabenzuständigkeit übernehmen würde. Aus der Sicht der Länder und ihrer Kommunen dürfte dies jedoch allenfalls dann interessant sein, wenn der Bund zugleich auch die Kosten übernimmt und keine Kompensation an anderer Stelle (z.B. bei der Verteilung der Umsatzsteuer) verlangt. Auch von daher sind erhebliche Zweifel an der Praktikabilität einer konkreten Umsetzung des geplanten Bundesteilhabegesetzes für die Eingliederungshilfe erlaubt.

Zwar lässt sich für die Gesamtheit der Flächenländer eine Abhängigkeit zwischen der Eingliederungshilfe, der sonstigen Sozialhilfe und der Jugendhilfe zur Quote der Arbeitslosen statistisch nicht belegen, aber es gibt einen sehr starken negativen Zusammenhang der Ausgaben für die Sozialleistungen mit den Ausgaben für Sachinvestitionen. Das Bestimmtheitsmaß von 69,4 \% ist hochsignifikant. $\mathrm{Ob}$ es sich dabei nur um eine Korrelation oder sogar um eine Regression handelt, mag an dieser Stelle dahin gestellt sein. In jedem Fall ist dieser Zusammenhang äußerst bemerkenswert. 
Abbildung 4: Verfügte und Verfügbare Finanzmasse nach Zinsen, Versorgung, Sachinvestitionen und Sozialleistungen im Jahr 2013 in Euro/E

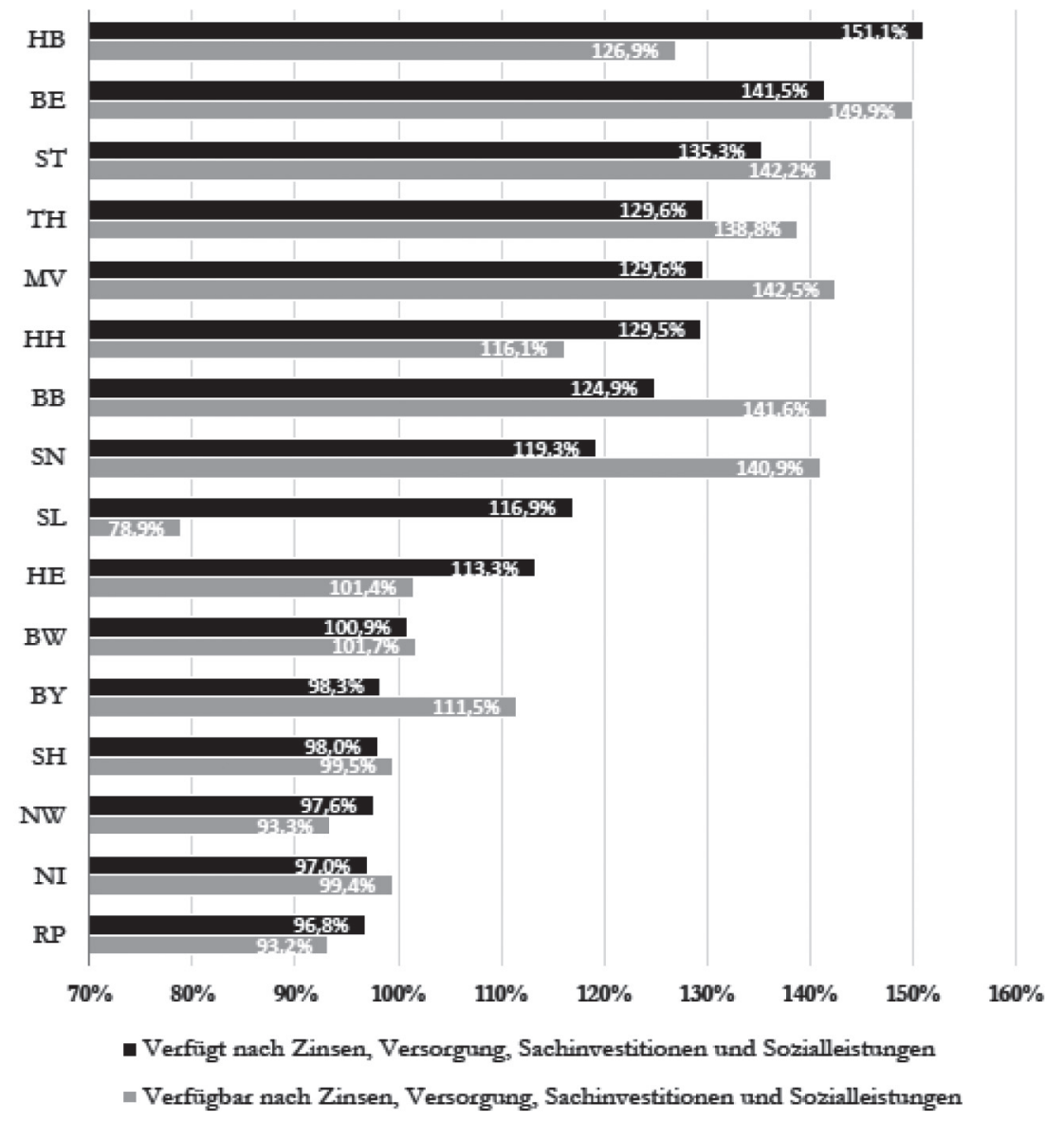

Quellen: Statistisches Bundesamt (Fachserie 14 Reihe 2 und Fachserie 13 Reihe 2.1), Bundesagentur für Arbeit (Grundsicherung für Arbeitssuchende - SGB II, Kosten der Unterkunft) und eigene Berechnungen

Jedenfalls ist es sehr auffällig, dass die Haushaltsprobleme insbesondere der Länder Bremen und Saarland zwar durch die Altlasten und hohe Sozialleistungen erheblich verstärkt werden, aber im Kern vor allem darauf beruhen, dass auch die nach Zinsen, Versorgung, Sachinvestitionen und Sozialleistungen verfügten Finanzmassen noch weit oberhalb der jeweiligen Vergleichsländer liegen. 
Bremen nimmt auch in dieser Abgrenzung mit 151,1\% des Durchschnitts der alten Flächenländer erheblich höhere Mittel in Anspruch als Berlin mit 141,5 \% und Hamburg mit 135,3 \%. Das Saarland benötigt nach Zinsen, Versorgung, Sachinvestitionen und Sozialleistungen mit 116,9\% des Durchschnitts der alten Flächenländer eine erheblich höhere Finanzmasse als die beiden sparsamsten Länder Rheinland-Pfalz mit 96,8 \% und Niedersachsen mit 97.0 \%. Auch Nordrhein-Westfalen mit 97,6 \%, Schleswig-Holstein mit 98,0 \%, Bayern mit 98,3\% und Baden-Württemberg mit 100,9 \% sind erheblich sparsamer als das Saarland. Unter den alten Flächenländern kommt in dieser Abgrenzung lediglich Hessen mit 113,3 \% auf einen Wert, der nur um 3,6 Punkte unter dem des Saarlandes liegt.

Noch weit vor dem Saarland positionieren sich allerdings die fünf neuen Flächenländer mit 119,3\% in Sachsen, 124,9 \% in Brandenburg, jeweils 129,6 \% in Mecklenburg-Vorpommern und in Thüringen und sogar 135,3\% in SachsenAnhalt.

Im Gegensatz zu Bremen und Hamburg sowie zu Hessen und dem Saarland, aber auch zu Rheinland-Pfalz, Nordrhein-Westfalen und Baden-Württemberg, liegen die verfügbaren Mittel in den neuen Ländern jedoch deutlich über den verfügten Mitteln. In Sachsen beträgt der Abstand 21,6 Punkte, in Brandenburg 16,7 Punkte, in Mecklenburg-Vorpommern 12,9 Punkte, in Thüringen 9,2 Punkte und selbst in Sachsen-Anhalt noch 6,9 Punkte.

Die neuen Länder können sich diese extrem hohe Inanspruchnahme von Finanzmasse nach Zinsen, Versorgung, Sachinvestitionen und Sozialleistungen z.Zt. also noch durchaus erlauben. Es stellt sich allerdings zum einen die Frage, ob diese Haushaltsstrukturen in nachhaltiger Weise mit den Zielen des Solidarpakts zu vereinbaren sind und - unmittelbar damit zusammenhängend - die noch wichtigere Frage, ob und welche Anpassungen in den neuen Flächenländern bis zum Jahr 2020 erforderlich sind, um auch dann noch die Vorgaben der Schuldenbremse einhalten zu können.

Wie stark sich z. Zt. noch die Haushaltsstrukturen der neuen von denen der alten Länder unterscheiden, lässt sich anhand der Abbildung 5 veranschaulichen. Hier werden für drei Kennziffern, nämlich die verfügbare Finanzmasse, die Sachinvestitionen und die nach Zinsen, Versorgung, Sachinvestitionen und Sozialleistungen in Anspruch genommene Finanzmasse, die Unterschiede zum Durchschnitt der alten Flächenländer in Euro/E dargestellt. Neben den Werten für die 
einzelnen neuen Flächenländer sind dabei auch die jeweiligen Durchschnitte für die neuen Flächenländer ausgewiesen.

Abbildung 5: Differenzen in Euro/E der neuen Flächenländer in 2013 gegenüber dem Durchschnitt der alten Flächenländer

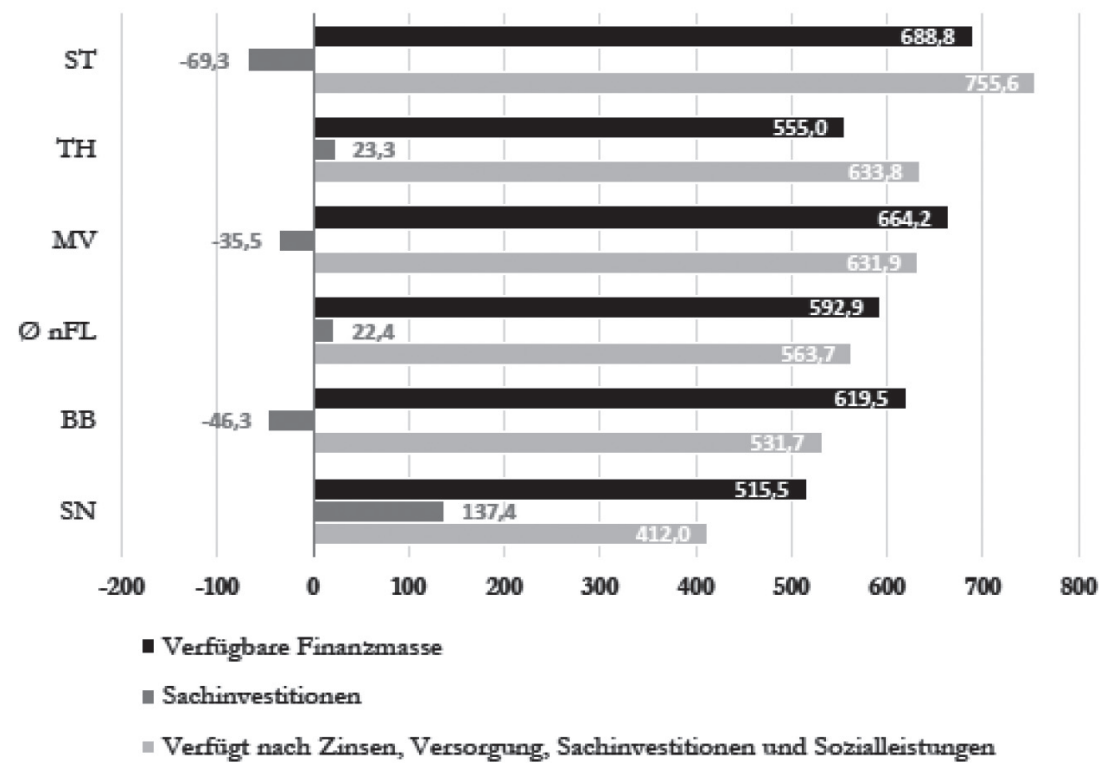

Quellen: Statistisches Bundesamt (Fachserie 14 Reihe 2 und Fachserie 13 Reihe 2.1), Bundesagentur für Arbeit (Grundsicherung für Arbeitssuchende - SGB II, Kosten der Unterkunft) und eigene Berechnungen

Die verfügbare Finanzmasse liegt aufgrund der Auswirkungen des Solidarpakts II und weiterer überproportionaler Leistungen für die neuen Länder (z.B. durch die SoBEZ aufgrund struktureller Arbeitslosigkeit und erheblich höherer Regionalisierungsmittel) zwischen 516 Euro/E in Sachsen und 689 Euro/E in SachsenAnhalt über dem Durchschnitt der alten Flächenländer (im Durchschnitt der neuen Flächenländer: 593 Euro/E = 14,1 \%).

Nach der Intention des Solidarpakts II sollen die überproportionalen Mittel für die neuen Länder vor allem zur Schließung der Infrastrukturlücke und zum Ausgleich der unterproportionalen kommunalen Finanzkraft sowie der Finanzierung von Sonderlasten aus der höheren strukturellen Arbeitslosigkeit dienen. Da hier eine konsolidierte Betrachtung der Finanzen der Länder und ihrer Kommunen erfolgt und die Sozialausgaben der neuen Länder in 2013 mit 803 Euro/E bereits 
unterhalb der Ausgaben der alten Flächenländer von 838 Euro/E lagen, sollten die überproportionalen Mittel eigentlich vollständig für überproportionale Investitionen verwendet werden.

Dies ist jedoch nicht der Fall. Bei den Sachinvestitionen liegen die Unterschiede zu den alten Flächenländern zwischen -69 Euro/E in Sachsen-Anhalt und +137 Euro/E in Sachsen (Durchschnitt: +22 Euro/E = 5,4 \%). Selbst im finanzpolitischen Musterland Sachsen werden also nur 26,7 \% und in Thüringen 4,2 \% der überproportionalen Finanzmasse für Sachinvestitionen verwendet. In Sachsen-Anhalt, Brandenburg und Mecklenburg-Vorpommern werden sogar weniger Mittel für Sachinvestitionen eingesetzt als im Durchschnitt der alten Flächenländer.

Dagegen liegen die nach Zinsen, Versorgung, Sachinvestitionen und Sozialleistungen in Anspruch genommene Finanzmassen zwischen 412 Euro/E in Sachsen und 756 Euro/ $\mathrm{E}$ in Sachsen-Anhalt höher als in den alten Flächenländern (Durchschnitt: 564 Euro/E = 26,4 \%). Es darf erheblich bezweifelt werden, dass sich dieser eindeutige Befund mit dem erklärten Ziel einer zügigen Schließung der Infrastrukturlücke vereinbaren lässt.

Die Ursache für diese offenkundige Fehlleitung der Mittel liegt darin, dass als Hauptkriterium im Solidarpakt II lediglich festgelegt wurde, dass die Summe aus den Investitionen und dem Ausgleich unterproportionaler kommunaler Steuerkraft mindestens dem Umfang der überproportionalen Mittel auf der Länderebene entsprechen sollte. Es wäre jedoch für einen möglichst schnellen Abbau der Infrastrukturunterschiede notwendig gewesen, eine verbindliche Zweckbindung der überproportionalen Mittel der Länderebene für überproportionale Investitionen sowie den Ausgleich unterproportionaler kommunaler Steuerkraft vorzusehen.

Insbesondere Sachsen-Anhalt, Thüringen und wohl auch Mecklenburg-Vorpommern werden deshalb aus heutiger Sicht die Schuldenbremse nur bei einem massiven Abbau der für die übrigen nichtinvestiven Verwendungen eingesetzten Mittel einhalten können. Die aktuellen Diskussionen in diesen Ländern zeigen allerdings, dass es hiergegen zunehmende politische Widerstände gibt.

\section{Die Finanzen der Länder und ihrer Kommunen im Jahr 2020 bei Fortführung des geltenden föderalen Finanzausgleichs}

Ob alle 16 Länder und ihre Kommunen im Zieljahr 2020 die Schuldenbremse einhalten können, d.h. ihre in Anspruch genommenen Finanzmassen nicht höher 
als ihre verfügbaren Finanzmassen ausfallen, hängt von einer ganzen Reihe von Faktoren bzw. Entwicklungen ab.

Um diese Frage fundiert zu beantworten, kommt es vor allem darauf an, möglichst realitätsnahe Projektionen für die Rahmenbedingungen des Jahres 2020 vorzunehmen. Folgende Annahmen werden dazu getroffen:

Die Projektion der Steuereinnahmen der jeweiligen Gesamtheit der Länder und ihrer Gemeinden erfolgt entsprechend der Steuerschätzung vom Mai 2014 mit einer unterstellten Fortschreibung in den Jahren 2019 und 2020 mit Zuwächsen von jeweils $3 \%$. Für die Regionalisierung dieser Steuern wird unterstellt, dass sich die Trends der Steuerkraftanteile der einzelnen Länder bzw. ihrer Gemeinden auf der Basis der Jahre 2005 -2013 bis zum Jahr 2020 linear fortsetzen. Zur Abschätzung der Differenzen zwischen den Realsteuereinnahmen und der Realsteuerkraft wird unterstellt, dass das Hebesatzgefälle zwischen den Gemeinden der einzelnen Länder des Jahres 2012 bestehen bleibt. Die gleiche Annahme wird für die Länder im Bereich der Grunderwerbsteuer getroffen. Im Risikoszenario wird unterstellt, dass die jährlichen Zuwächse der Steuereinnahmen um jeweils einen Punkt geringer ausfallen als in der Standardprojektion. Auf ein zusätzliches Chancenszenario wird in Anbetracht der aktuellen "Schönwetterprojektion“ für die gesamtwirtschaftliche Entwicklung im Rahmen der Steuerschätzung vom Mai 2014 verzichtet.

Die Projektion der Einwohnerzahlen der einzelnen Länder erfolgt auf der Basis der nach dem Zensus fortgeschriebenen Einwohnerzahl vom 30.6.2013 und der Variante W1 der 12. koordinierten Vorausberechnung. Anstatt des in der Variante W1 unterstellten Wanderungsüberschusses von jährlich 100.000 wird jedoch aufgrund der Entwicklung der letzten Jahre mit einem jährlichem Wanderungsüberschuss von 300.000 kalkuliert, wobei der zusätzliche jährliche Überschuss von 200.000 auf die Länder entsprechend ihrer Nettozuzugsanteile des Jahres 2013 verteilt werden.

Eine möglichst genaue Projektion der Einwohnerzahlen ist schon deshalb notwendig, weil die Einwohnerzahl die entscheidende Stellgröße im föderalen Finanzausgleich ist. Für diesen wird zunächst einmal unterstellt, dass sich die Verteilungsregeln auch nach dem Auslaufen des Maßstäbe- und des Finanzausgleichsgesetzes nicht verändern. Einzige Ausnahme stellen die SoBEZ für strukturelle AL dar. Hier wird eine Absenkung der Zusatzförderung der neuen Länder auf 400 Mio. Euro unterstellt. $\mathrm{Zu}$ den jetzigen Regeln gehört auch ein völliges und ersatzloses Auslaufen der teilungsbedingten SoBEZ. 
Desgleichen wird unterstellt, dass die überproportionalen Bundesleistungen für die neuen Länder aus dem Korb II ersatzlos fortfallen. Für die sonstigen Zahlungen des Bundes an die 16 Länder wird unterstellt, dass sie unverändert bleiben und in Niveau und Verteilung den Ergebnissen des Jahres 2013 entsprechen. Seitens der EU wird davon ausgegangen, dass die überproportionale Förderung für die neuen Länder nochmals deutlich auf $31 \%$ des Niveaus der Förderperiode 2007 - 2013 reduziert wird und im Übrigen ein jährlicher Zuwachs der Zahlungen durch die EU um $1 \%$ erfolgt.

Zur Abschätzung der Schuldenstände zum 31.12.2019 wird in dieser Projektion für alle Länder und ihre Kommunen unterstellt, dass ihre um die Salden der finanziellen Transaktionen bereinigten Finanzierungssalden des Jahres 2013 (positive und negative) bis zum Jahr 2020 in sieben gleichen Schritten auf null abgebaut werden. Die Werte des Basisjahrs 2013 werden dabei zusätzlich um die Unterschiede zwischen den tatsächlichen und den periodengerechten Finanzausgleichsleistungen korrigiert. Für die Zinsbelastung des Jahres 2020 wird ein durchschnittlicher Zinssatz von 2,75\% unterstellt. Im Risikoszenario wird ein Anstieg des Zinsniveaus auf 3,5\% und im Chancenszenario eine Absenkung auf $2 \% \mathrm{zu}$ Grunde gelegt. Das Chancenszenario ist allerdings wohl nur dann zu erwarten, wenn die Zinsen weltweit - d.h. insbesondere auch in den USA - dauerhaft niedrig bleiben, was wohl nur bei einer schwachen wirtschaftlichen Entwicklung mit geringeren Steuereinnahmen zu erwarten sein dürfte.

Die Projektion der Ausgaben für Versorgung, Beihilfe der Versorgungsempfänger und (bei den neuen Ländern einschließlich Berlin) aus dem AAÜG beruht im Wesentlichen auf den um die (geringen) Abweichungen des Jahres 2013 korrigierten Berechnungen von Benz et al. ${ }^{6}$, wobei durchschnittliche Erhöhungen der Versorgungsbezüge um jährlich 2,5\% (anstatt von $2 \%$ bei Benz et al.) unterstellt werden. Die Zuwächse der Ausgaben nach dem AAÜG orientieren sich an den mittelfristigen Planungen der neuen Länder.

6 Benz, T./Hagist, Chr./Raffelhüschen, B.: Ausgabenprojektion und Reformszenarien der Beamtenversorgung in Deutschland, Berlin, 2011, Anhang V, Tabelle 13. 
Abbildung 6: Summen aus Zinsen und Versorgung als Anteile der verfügbaren Finanzmassen in 2013 und 2020

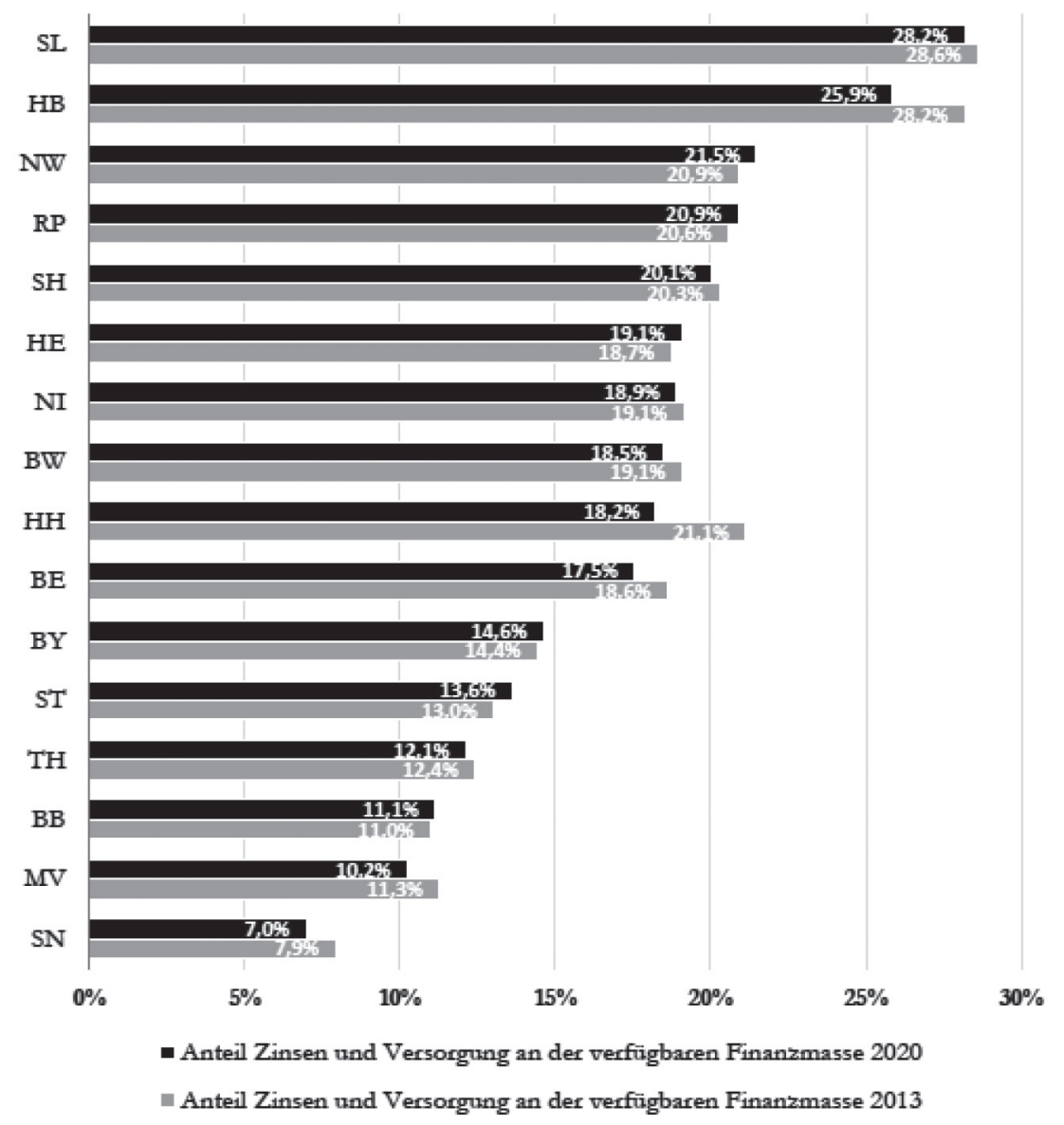

Quellen: Statistisches Bundesamt (Fachserie 14 Reihe 2 - 2013, Fachserie 14 Reihe 10.1 - 2013, Vorläufige Wanderungsergebnisse 2013, 12. koordinierte Bevölkerungsvorausberechnung, Bevölkerungsstand 30.06.2013,), Bundesministerium der Finanzen (Abrechnungen der Finanzausgleiche 2005 - 2012), Bundesratsdrucksache 425/14 (Abrechnung des Finanzausgleichs 2013), Benz et al., a.a.O. und eigene Berechnungen

Es zeigt sich, dass die Möglichkeiten zur Einhaltung der Schuldenbremse im Jahr 2020 vor allem dadurch auseinanderdriften, dass die „Altlasten“ aus Zinsen und Versorgung sehr unterschiedlich verteilt sind und bleiben. Die fünf neuen Länder müssen lediglich einen relativ kleinen Anteil ihrer verfügbaren Finanzmasse für 
Zinsen und Versorgung aufwenden, nämlich zwischen 7,0\% in Sachsen und $13,6 \%$ in Sachsen-Anhalt. Demgegenüber müssen Bremen 25,9 \% und das Saarland sogar 28,2 \% für Zinsen und Versorgung ausgeben.

Aus der Abbildung 7 lässt sich erkennen, dass die bis zum Jahr 2020 für die einzelnen Länder und ihre Kommunen zu erfüllenden Konsolidierungsaufgaben zum einen von den bei Einhaltung der Schuldenbremse verfügbaren Mitteln pro Einwohner nach Zinsen und Versorgung abhängen, zum anderen jedoch vor allem von der Differenz aus den verfügten Mitteln nach Zinsen und Versorgung in 2013 und den verfügbaren Mitteln in 2020. ${ }^{7}$

Das Saarland steht dabei vor einer nahezu unlösbaren Aufgabe. Nach Zinsen und Versorgung verfügt das Land in 2013 mit 110,6 \% über weit überproportionale Mittel und müsste in 2020 mit $84 \%$ des Durchschnitts der alten Flächenländer auskommen. Auch das Land Bremen dürfte größte Probleme mit der Einhaltung der Schuldenbremse haben. In 2013 hat das Land über 142,0 \% verfügt und müsste die Inanspruchnahme von Mitteln bis 2020 auf 120,3 \% reduzieren. Dies dürfte für den Stadtstaat Bremen mit jetzt schon äußerst niedrigen Sachinvestitionen und recht hohen Sozialausgaben nahezu ausgeschlossen sein.

Ganz anders - aber auch nicht gerade einfach - stellen sich die zu lösenden Aufgaben in den fünf neuen Ländern und in Hessen dar. Denn allen sechs Ländern dürften 2020 nach Zinsen und Versorgung deutlich höhere Finanzmassen zur Verfügung stehen als dem Durchschnitt der alten Flächenländer, wobei das Spektrum zwischen 102,6 \% in Hessen und 110,5 \% in Sachsen reicht. Dennoch müssen alle sechs Länder bis 2020 erheblich stärker konsolidieren als der Durchschnitt der alten Flächenländer. Insbesondere Sachsen-Anhalt (von 120,4 \% auf 105,2 \%), Thüringen (von 116,4 \% auf 104,3 \%) und Hessen (von 111,9\% auf $102,6 \%$ müssen zur Einhaltung der Schuldenbremse bis 2020 noch gewaltige Konsolidierungsanstrengungen unternehmen.

7 Durch Division der Prozentsätze der relativen Verfügbarkeit nach Zinsen und Versorgung im Jahr 2020 und der relativen Inanspruchnahme im Jahr 2013 erhält man den sogenannten $P w C$ Nachhaltigkeitsindex. Vgl.: Detemple, P. et al: a.a.O., hier $185 \mathrm{ff}$. 
Abbildung 7: Verfügbare Finanzmasse in 2020 und verfügte Finanzmasse in 2013 (jeweils in Relation zum Durchschnitt der alten Flächenländer nach Zinsen und Versorgung)

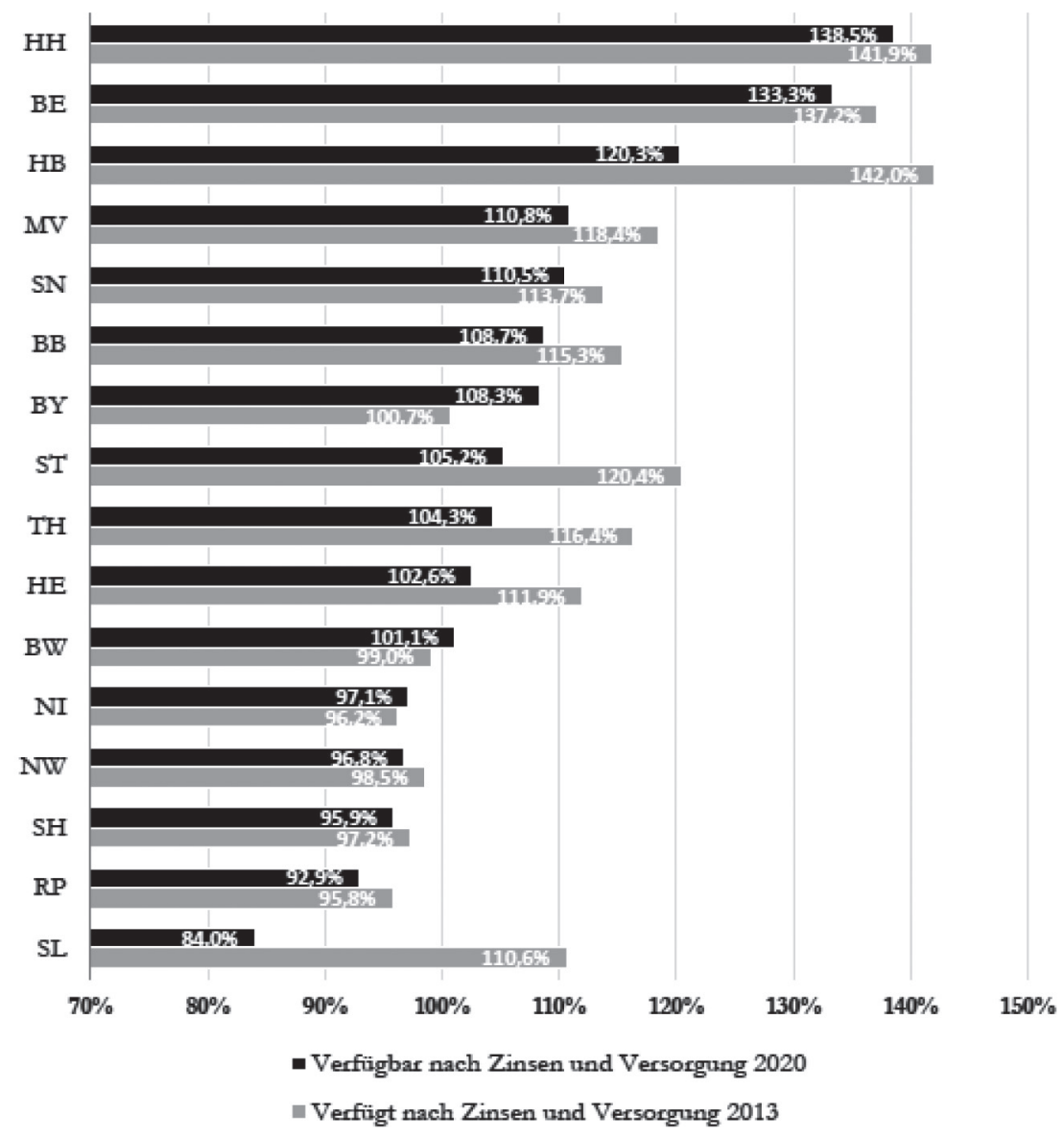

Quellen: Statistisches Bundesamt (Fachserie 14 Reihe 2 - 2013, Fachserie 14 Reihe 10.1 - 2013, Vorläufige Wanderungsergebnisse 2013, 12. koordinierte Bevölkerungsvorausberechnung, Bevölkerungsstand 30.06.2013), Bundesministerium der Finanzen (Abrechnungen der Finanzausgleiche 2005 - 2012), Bundesratsdrucksache 425/14 (Abrechnung des Finanzausgleichs 2013), Benz et al., a.a.O. und eigene Berechnungen

Lediglich Sachsen (von 113,9\% auf 110,5\%) ist auf einem sehr guten und Brandenburg (von 115,3\% auf 108,7 \%) sowie Mecklenburg-Vorpommern (von $118,4 \%$ auf $110,8 \%$ ) sind auf einem guten Konsolidierungskurs. Sehr bemer- 
kenswert ist dabei vor allem, dass Mecklenburg-Vorpommern, Sachsen und Brandenburg aufgrund ihrer sehr niedrigen Belastungen mit Versorgungsausgaben im Jahr 2020 nach Zinsen und Versorgung sogar über noch höhere Finanzmassen verfügen können als Bayern.

Noch anders stellen sich die Probleme in Rheinland-Pfalz (von 95,8\% auf 92,9\%), Schleswig-Holstein (von 97,2 \% auf 95,9\%) und Nordrhein-Westfalen (von 98,5 \% auf 96,8 \%) dar. Diese drei Länder haben nämlich bereits in 2013 unterdurchschnittliche Mittel in Anspruch genommen, müssen jedoch dennoch bis 2020 noch stärker konsolidieren als der Durchschnitt. Insbesondere in Nordrhein-Westfalen kommt erschwerend hinzu, dass die Sachinvestitionen schon jetzt extrem niedrig, die Sozialausgaben sehr hoch und die kommunalen Hebesätze für die Realsteuern sogar extrem hoch sind. ${ }^{8}$

Dass es jedoch durchaus möglich ist, den Haushalt auch dann noch auszugleichen, wenn die verfügbaren Mittel unterhalb des Durchschnitts liegen, beweist insbesondere Niedersachsen (von 96,2\% auf 97,1\%).

Bayern (von 100,7 \% auf 108,3 \%) dürfte keinerlei Probleme mit der Schuldenbremse haben und auch Baden-Württemberg (von 99,0 \% auf 101,1\%) dürfte die Schuldenbremse ohne größere zusätzliche Konsolidierungsmaßnahmen einhalten können.

Deutlich anspruchsvoller ist dagegen die Ausgangssituation in Berlin (von $137,2 \%$ auf 133,3\%). Dies liegt vor allem daran, dass die Sachinvestitionen äußerst niedrig und andererseits die Sozialleistungen extrem hoch liegen. Zugleich wird - für jeden Besucher in Berlin leicht erkennbar - offenkundig auch die Bau- und Straßenunterhaltung erheblich vernachlässigt. Es ist deshalb fraglich, ob und wie lange das Land einen solchen Kurs beibehalten kann. Dennoch sind die Konsolidierungserfolge des letzten Jahrzehnts durchaus beeindruckend. Hinzu kommt, dass die Einwohnerzahlen der Hauptstadt auch weiterhin kräftig zunehmen dürften, was den Konsolidierungsdruck deutlich reduziert.

Auch Hamburg (von 141,9 \% auf 138,5 \%) muss noch ein Stück konsolidieren, kann aber im Vergleich zu Berlin über eine deutlich höhere Finanzmasse verfügen, hat geringere Sozialbelastungen und investiert auf einem sehr hohen Niveau.

8 Nach dem Realsteuervergleich 2013 lag der durchschnittliche gewichtete Hebesatz der Gemeinden in Nordrhein-Westfalen (Grundsteuer A, Grundsteuer B und Gewerbesteuer) bei 454\%, im Durchschnitt der übrigen Flächenländer dagegen nur bei $379 \%$. 
$\mathrm{Zu}$ berücksichtigen ist allerdings auch, dass es sich bei den Werten in Abbildung 7 nicht um absolute, sondern um relative Werte pro Einwohner handelt. Entwickelt sich die Einwohnerzahl unterdurchschnittlich, wie z.B. im Saarland und in den neuen Ländern, so entstehen zusätzliche Konsolidierungserfordernisse. Bei überdurchschnittlicher Einwohnerentwicklung, wie z.B. in Hamburg, Berlin, Baden-Württemberg oder Bayern, verringern sich dagegen die Konsolidierungsnotwendigkeiten.

Insofern ist es sinnvoll, auch die absoluten Konsolidierungspfade zu kalkulieren. Sie geben an, wie stark die in Anspruch genommene gesamte Finanzmasse nach Zinsen und Versorgung in den einzelnen Ländern zwischen 2013 und 2020 jährlich zunehmen darf, damit in 2020 die Schuldenbremse eingehalten wird.

Dabei ist es sinnvoll, nicht von nominalen, sondern von realen Werten auszugehen. Zur Berechnung der realen Konsolidierungspfade reicht es allerdings nicht aus, die Zuwachsraten nur um die allgemeine Preissteigerungsrate zu bereinigen. Da bei den Ländern und ihren Kommunen ein Großteil der Finanzmasse nach Zinsen und Versorgung für eigene Personalkosten oder Zuschüsse zu Personalkosten bei Dritten aufgewendet wird, ergeben sich wegen der im Durchschnitt relativ geringen Effizienzsteigerungen im öffentlichen Bereich Kostensteigerungen, die längerfristig gesehen etwa 0,5 Punkte oberhalb des Anstiegs der Verbraucherpreise liegen. Kalkuliert man mit einem durchschnittlichen Anstieg der Verbraucherpreise im Zeitraum von 2013 - 2020 von 1,5\%, so ist dementsprechend für den öffentlichen Bereich mit jährlichen Kostensteigerungen von rund $2 \%$ zu rechnen.

In der Abbildung 8 ist für das Jahr 2020 mit drei unterschiedlichen Zinsentwicklungen kalkuliert worden. Im Normalszenario wird ein Zinsniveau von 2,75 \%, im Risikoszenario eines von 3,5\% und im Chancenszenario eines von $2 \%$ unterstellt. 
Abbildung 8: Zur Einhaltung der Schuldenbremse zulässige jährliche reale Steigerung des Einsatzes an Finanzmasse nach Zinsen und Versorgung zwischen 2013 und 2020 bei alternativen Zinsniveaus in 2010.

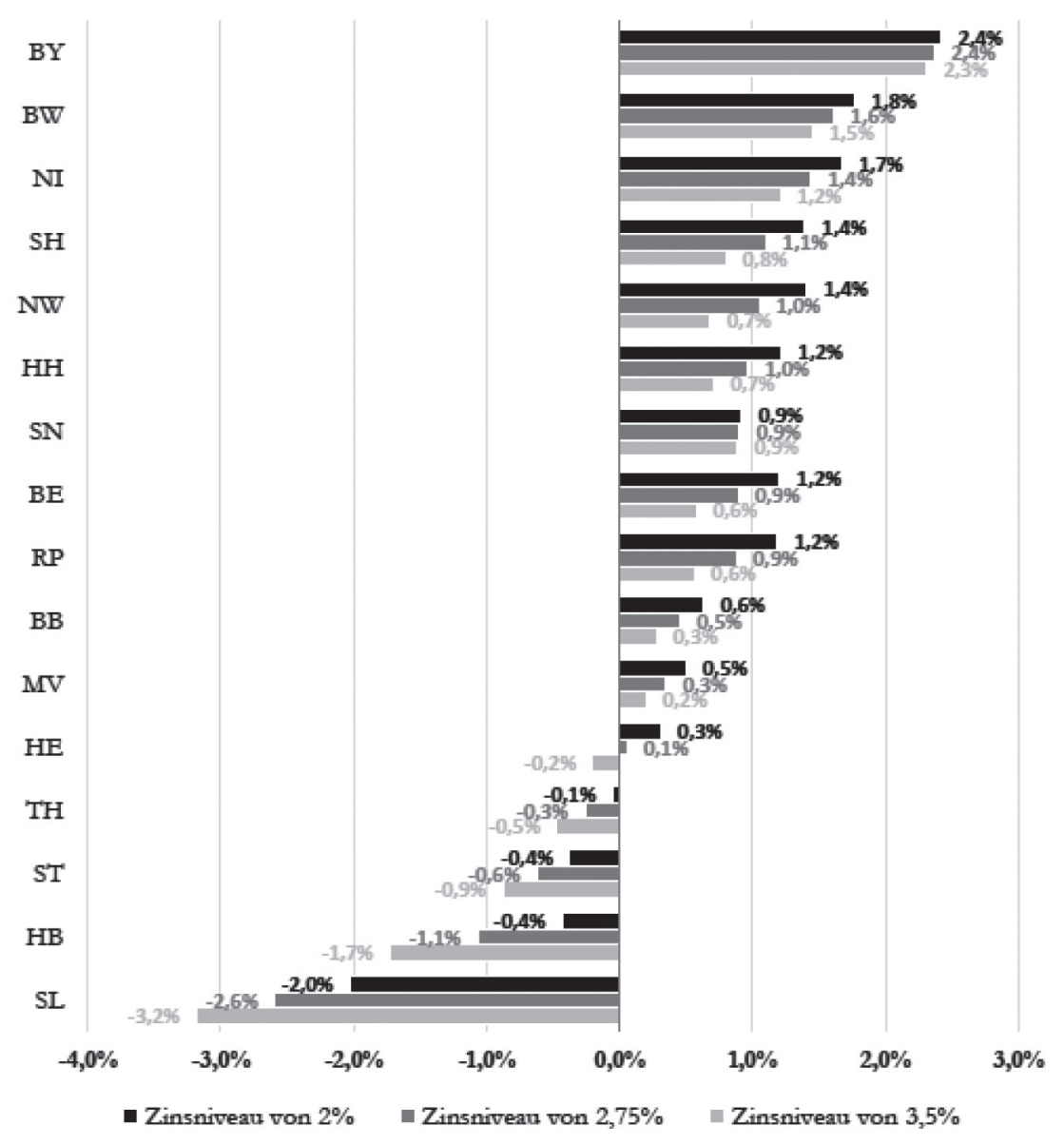

Quellen: Statistisches Bundesamt (Fachserie 14 Reihe 2 - 2013, Fachserie 14 Reihe 10.1 - 2013, Vorläufige Wanderungsergebnisse 2013, 12. koordinierte Bevölkerungsvorausberechnung, Bevölkerungsstand 30.06.2013), Bundesministerium der Finanzen (Abrechnungen der Finanzausgleiche 2005 - 2012), Bundesratsdrucksache 425/14 (Abrechnung des Finanzausgleichs 2013), Benz et al., a.a.O. und eigene Berechnungen 
Erwartungsgemäß wirken sich unterschiedliche Zinsniveaus besonders stark bei den überdurchschnittlich verschuldeten Ländern Saarland und Bremen aus, während die alternativen Konsolidierungspfade in Sachsen und Bayern fast identisch sind.

Sollte dieses Risikoszenario Realität werden, dürfte es dem Saarland völlig unmöglich werden die Schuldenbremse einzuhalten. Eine jährliche reale Einsparung von 3,2 \% ist ausgeschlossen. Auch Bremen dürfte es im Risikoszenario bei dann notwendigen jährlichen realen Einsparungen von 1,7 \% nahezu unmöglich werden, Haushalte ohne neue Schulden aufzustellen. Aber auch in den Ländern Sachsen-Anhalt (- 0,9\%), Thüringen (- 0,5\%) und Hessen (- 0,2 \%) wären in diesem Szenario erhebliche reale Einsparungen notwendig.

Dabei ist zusätzlich zu beachten, dass insbesondere im Bereich der U3Betreuung und der sozialen Leistungen weiter steigende Haushaltsbelastungen programmiert sind, so dass die übrigen Aufgabenbereiche noch mehr unter Konsolidierungsdruck geraten.

Der gleichen Entwicklung bei der U3-Betreuung und den sozialen Leistungen sind auch die elf Länder (einschließlich ihrer Kommunen) ausgesetzt, die auch im Risikoszenario noch reale Zuwächse verausgaben können. Insbesondere in Mecklenburg-Vorpommern, Brandenburg, Rheinland-Pfalz, Berlin, Hamburg, Nordrhein-Westfalen und Schleswig-Holstein sind deshalb - zumindest im Risikoszenario - weitere Konsolidierungsanstrengungen unabweisbar. Lediglich Sachsen, Niedersachsen, Baden-Württemberg und Bayern dürften auch im Risikoszenario ihre Haushalte ohne weitere tiefgreifende Konsolidierungsmaßnahmen ausgleichen können.

Noch stärkere Konsolidierungserfordernisse ergeben sich bei einer Absenkung der jährlichen Zuwächse der Steuereinnahmen gegenüber dem Grundmodell um jeweils einen Punkt. Danach müssten bis auf Bayern, Baden-Württemberg und Niedersachsen alle anderen Länder real konsolidieren, wobei es dann erst recht für das Saarland und Bremen unmöglich werden dürfte, die Schuldenbremse einzuhalten, aber auch die anderen elf Länder müssten ihre Konsolidierungsbemühungen zur Einhaltung der Schuldenbremse nochmals deutlich verstärken. 
Abbildung 9: Zur Einhaltung der Schuldenbremse zulässige jährliche reale Steigerung des Einsatzes an Finanzmasse nach Zinsen und Versorgung zwischen 2013 und 2020 bei alternativen Wachstumsraten der Steuereinnahmen bis zum Jahr 2020

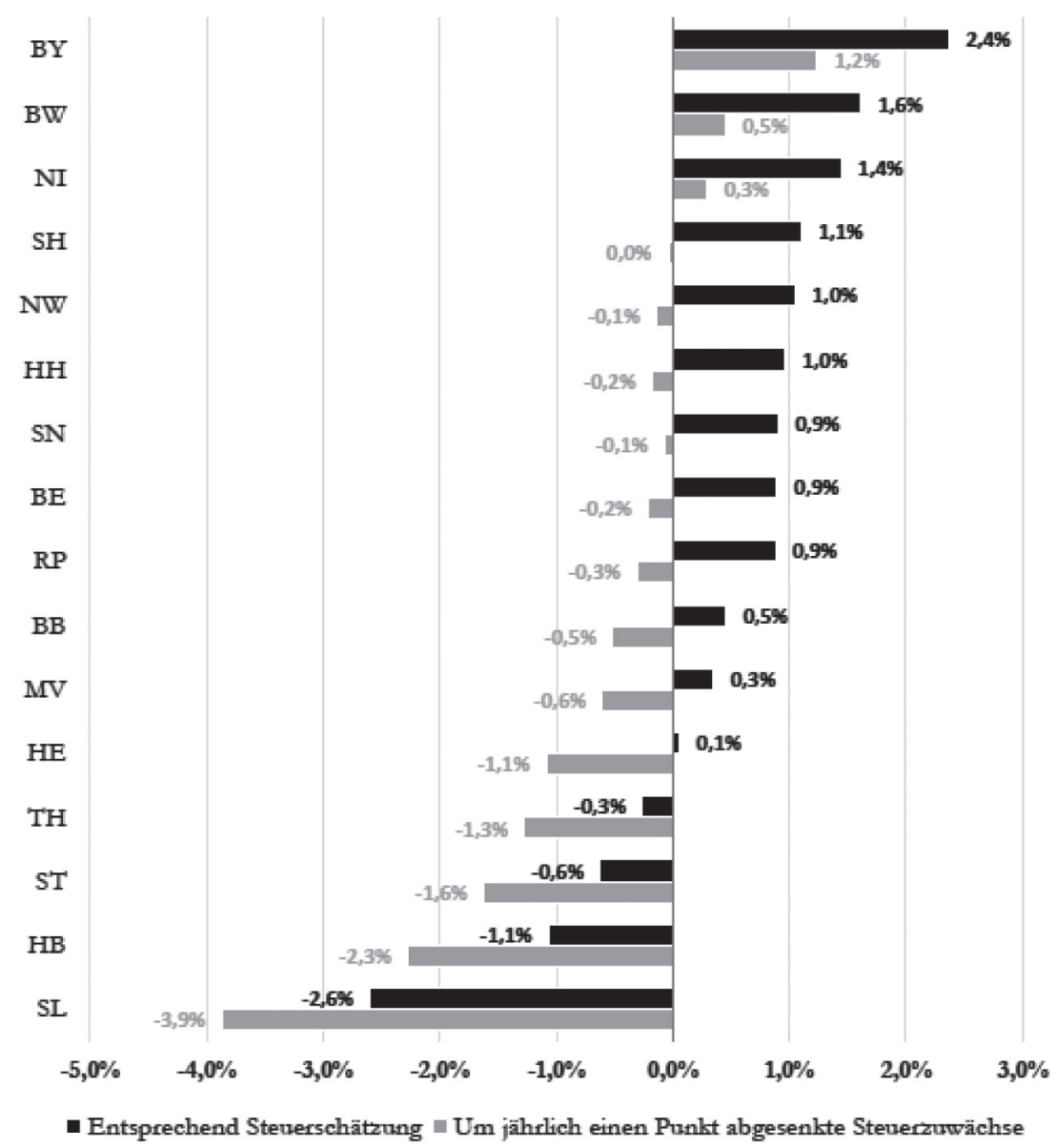

Quelle: Statistisches Bundesamt (Fachserie 14 Reihe 2 - 2013, Fachserie 14 Reihe 10.1 2013, Vorläufige Wanderungsergebnisse 2013, 12. koordinierte Bevölkerungsvorausberechnung, Bevölkerungsstand 30.06.2013), Bundesministerium der Finanzen (Abrechnungen der Finanzausgleiche 2005 - 2012), Bundesratsdrucksache 425/14 (Abrechnung des Finanzausgleichs 2013), Benz et al., a.a.O. und eigene Berechnungen 


\section{Zusammenfassung und Ausblick}

In den nächsten Jahren werden die Weichen für die Fortentwicklung der Finanzverfassung gestellt. Dabei ist darauf zu achten, dass mögliche alle oder zumindest fast alle Länder und ihre Kommunen in die Lage versetzt werden, spätestens ab dem Jahr 2020 ihre Haushalte strukturell auszugleichen. Eine differenzierte Analyse auf der Basis der geltenden Finanzausgleichsregeln zeigt, dass bei einer normalen wirtschaftlichen Entwicklung bis auf das Saarland und Bremen alle Länder dieses Ziel erreichen können, wobei in einzelnen Ländern allerdings noch erhebliche Konsolidierungsanstrengungen erforderlich sind.

Sollten allerdings die Zinsen bis zum Jahr 2020 wieder kräftig ansteigen oder die Steuereinnahmen sehr viel langsamer zunehmen als durch die Steuerschätzung aus dem Mai 2014 projiziert, dürften nur wenige Länder ohne deutliche zusätzliche Konsolidierungsanstrengungen die Schuldenbremse einhalten können.

Eine ganz wichtige Voraussetzung für diese relativ positive Perspektive ist allerdings, dass der Finanzausgleich auch nach seiner notwendigen Neuordnung so leistungsfähig bleibt, dass steuer- und strukturschwächere Länder auch zukünftig nicht überfordert werden. Die vielfach geforderte generelle Absenkung der Ausgleichsintensität des Finanzausgleichs dürfte unter diesen Umständen mit der verfassungsrechtlichen Vorgabe ausgeglichener Haushalte kaum zu vereinbaren sein. Damit soll nicht einer Beibehaltung der jetzigen Struktur des föderalen Finanzausgleichs das Wort geredet werden. An anderer Stelle ist jedoch schon dargestellt worden, dass es sehr wohl möglich erscheint die systematischen Mängel des jetzigen Systems zu beseitigen und dennoch größere Verwerfungen bzw. Umverteilungen zu vermeiden. ${ }^{9}$

Ein auch weiterhin leistungsfähiger Finanzausgleich würde insbesondere die neuen Länder bei sparsamer Haushaltsführung in die Lage versetzen, auch noch nach dem Jahr 2019 Haushaltsüberschüsse zu erzielen oder zumindest die Haushalte auszugleichen. Bei einer Einführung von Hebesatz- oder Zuschlagsrechten auf den proportionalen Teil der Einkommensteuer und die Körperschaftsteuer müssten die neuen Länder somit ihre Steuersätze nicht etwa anheben, sondern könnten sie aufgrund ihrer geringeren Bemessungsgrundlagen besonders stark absenken und sich somit Standortvorteile verschaffen, die auf längere Sicht mit

9 Vgl. z.B.: Deubel, I.: Soziallastenberücksichtigung im Finanzausgleich und Lösung des Altschuldenproblems, in: Henneke, H.-G.: Gesicherte Kommunalfinanzen trotz Verschuldungs- und Finanzkrise, Schriften zum deutschen und europäischen Kommunalrecht, Band 46, Berlin 2014, 118 - 140. 
hoher Wahrscheinlichkeit deutlich wirksamer wären als ein alternativer Solidarpakt III.

Die Einführung einer aus föderaler Sicht wünschenswerten stärkeren Steuerautonomie dürfte allerdings politisch nur dann umsetzbar sein, wenn vor allem die steuerschwächeren Länder in die Lage versetzt würden, ihre Haushalte ohne die Erhebung überdurchschnittlicher Steuersätze auszugleichen. Solange steuerstarke Länder und Teile der Wissenschaft das Ziel propagieren, den Finanzausgleich deutlich zu reduzieren und stattdessen eine Steuerautonomie auf der Länderebene einzuführen, dürften im Bundesrat keine Mehrheiten für eine solche Steuerautonomie organisiert werden können. 